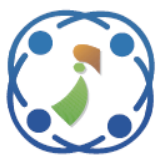

\title{
Hybrid Collaborative Movie Recommender System Using Clustering and Bat Optimization
}

\author{
Vimala Vellaichamy ${ }^{1 *}$ \\ Vivekanandan Kalimuthu ${ }^{1}$ \\ ${ }^{1}$ Department of Computer Science and Engineering, Pondicherry Engineering College, Pondicherry, India \\ * Corresponding author’s Email: vimsvickyvimal@gmail.com
}

\begin{abstract}
A Recommender system (RS) is an information filtering software that helps users with a personalized manner to recommend online products to Users and give suggestions about the products that he or she might like. In e-commerce, collaborative Movie recommender system assist users to select their favorite movies based on their similar neighbor's movie ratings. However due to data sparsity and scalability problems, neighborhood selection is more challenging with the rapid increasing number of users and movies. In this paper, a hybrid Collaborative Movie Recommender system is proposed that combines Fuzzy C Means clustering (FCM) with Bat optimization to reduce the scalability problem and enhance the clustering which improves recommendation quality. Fuzzy c means clustering is used to cluster the users into different groups. Bat Algorithm is used to obtain the initial position of clusters. Lastly, the proposed system creates movie recommendations for target users. The proposed system was evaluated over Movie Lens dataset. Experiment results obtained show that the proposed Algorithm can yield better recommendation results compared to other techniques in terms of Mean Absolute Error (MAE), precision and Recall.
\end{abstract}

Keywords: Recommender system, Collaborative filtering, Fuzzy c means clustering, Bat Algorithm.

\section{Introduction}

Recommender systems have increased wellknown acceptance during last years, leveling the ground for new sale opportunities in e commerce [1]. Recently, the internet has more amounts of digital information and the more number of users which creates information overload. This needs efficient information filtering to extract essential information from the huge amount of data. Recommendation systems have been developed to reduce the information overload problem. Recommender system works under the information filtering system [2].Recommender systems assist users to take a decision under complex situations in the search of interesting products among a massive data. It also makes a personalized recommendation according to user's preferences and interests. Now a day's Recommender System is very much useful in e-shopping, e-learning, e-tourism, e-government, ebusiness and a social networking such as Face Book. In e-shopping, RS recommend items such as Books, movies, music, news, research articles, CDs/DVDs, etc.to their customers. Recommender systems take different type of inputs such as explicit input and implicit input. The explicit is the rating given by users explicitly regarding their interests on the items. Implicit was derived from user behavior such as navigation and browsing history. Recommender systems can be mainly classified in to content-based, collaborative and hybrid recommender filtering techniques [3].Collaborative filtering $(\mathrm{CF})$ is the most traditional and commonly used approach to generate recommendations. Collaborative-filtering [4] identify neighboring users who have same interests and preferences in the past by calculating similarities between their profiles and recommend the products by computing a weighted average of these neighbor users' rating. For Example, Amazon shopping mart uses a collaborative recommendation method that provides suggestions about products to customers. It can scales up to tens of millions of customers and products in real time[5].Content Based Recommender System suggests items based 
on user choices made in the past [6].Content Based recommendation systems are used to recommend text documents like web pages and newsgroup messages. Fab is an example of content based recommender system [7]. Hybrid RS combines the collaborative filtering and content based approaches to get the advantages of each of them. The combination of different recommendation algorithms will provide more effective and accurate recommendations than a single recommendation algorithm .The disadvantage of one algorithm can be overcome by another algorithm [8]. The combinations of seven hybridization methods are used in hybrid recommender systems [9]. The methods are weighted, mixed, switching, feature combination, feature augmentation, cascade and meta level. Collaborative filtering is categorized into memory based and model based methods. [10]. Memory based method uses the complete data base to find the $\mathrm{k}$ nearest neighbors for a current user, and generate a number of recommendations to the user. Memory based CF has some limitations such as sparsity, poor scalability and cold start problem. Data sparsity occurs when only a few number of items are rated by users and so the users profile have more missing values. This leads to a sparse user rating matrix and unable to find more effective neighbors.so the system produce weak recommendations to users. Scalability occurs when Computation normally grows linearly with the number of customers and products. This increases the cost and thus reduces the performance of recommender system. Cold Start problem happens if Recommender system does not have sufficient data about a new user or new item to make relevant predictions. Model-based algorithms use the useritem dataset to learn a model in offline and store ratings which are then used to create predictions. This reduces the scalability and sparsity problems. The recommendation quality of model-based method is good as well as memory based ones. The learning process of model based is done by using data mining and machine learning techniques [11]. The techniques are Dimensionality reduction (PCAPrincipal Component Analysis), regression, clustering, association rule mining etc. Clustering and Association rule based methods are some popular techniques which are used under model based collaborative filtering. Clustering based $\mathrm{CF}$ give more accurate predictions for sparse data [12]. Most clustering-based $\mathrm{CF}$ methods use $K$-means clustering, but $\mathrm{K}$ means clustering assign data points (item/ user) to only one cluster and they are restricted to group items/users into multiple clusters. Fuzzy C-Means is a clustering method that permits each individual data can assigned to more than one cluster through different membership degree [13]. In this paper, a model based collaborative recommender system is proposed to reduce the data sparsity and scalability issues. We use fuzzy cmeans (FCM) clustering technique to partition the users into groups. But fuzzy-C means lacks the capability of choosing the initial cluster Centre point and affects the accuracy of clustering. The random initial cluster Centre selection can result in a local optimum solution. Bat Algorithm is employed as optimization method to find the initial cluster point that improves the performance of clustering. We also examine that the performance of Movie Lens data regarding with MAE, Precision and Recall. The experiment results indicates that the proposed method is more efficient and provide more reliable suggestions for products comparing with existing clustering based collaborative systems. Our proposed collaborative movie recommender system is used to achieve a personalized recommendations for a user while reduces the scalability issue. The rest of the paper is organized as follow. Section 2 discusses about the overview of the previous research studies on clustering based $\mathrm{CF}$ and addressing the challenges in CF. Section 3 introduces the framework and steps of the proposed algorithm. Section 4 presents experimental Results of proposed method .Section 5 finally draws conclusions.

\section{Related work}

Recommendation systems were first developed by Tapestry project in 1992. It is one of the most popular information management systems [14]. Collaborative filtering recommendation system is the most powerful method which is based on the nearest neighbor mechanism. The basic idea in CF is that to recommend a product to target user is to find other users who have similar past history rating pattern with him. Users who have similar past ratings might be on the maximum chances that have the same preference ratings in the future also. Rating of a product for a target user can be predicted by the weighted similarity of neighborhoods. Clustering is a process of partitioning the data points into a set of disjoint groups called clusters that aimed at minimizing the dissimilarity between data assigned to the same cluster. Clustering was used to quickly locate a user's neighbors. It is a promising way to improve the scalability of collaborative filtering by reducing the search for the Neighborhoods in the preference space, and generates some recommendations for users without using entire 
dataset. Birtolo, D. Ronca [15] proposed Item-based Fuzzy Clustering recommender system which locating users in suitable classes and providing recommendations with best clusters of users. This method increases the coverage of predictions. Treerattanapitak, Jaruskulchai [16] developed exponential fuzzy $\mathrm{C}$ means clustering based recommendation by changing the clustering's objective function with an exponential function so that it will enhance the membership assignment and perform well better than other Fuzzy C Means. Koohi, K.Kiani [17] employ a User Based Collaborative Filtering using Fuzzy C Means and its performance is evaluated against different clustering methods such as K-means, Self-Organizing Map (SOM). The limitation of the above methods [15-17] is, FCM is more sensitive to initialization and get stuck in local optima. This affects the clustering and also reduces the recommendation quality. Lia nd Kim use fuzzy K-means clustering technique to cluster the items and combined with the content information for similarity calculation to improve the accuracy recommendation [18]. It indicates that the proposed cluster based method solve the cold start problem. But it is not appropriate for large data sets and every object (user, item) can be assigned to only one cluster. Kim and Ahn's applied K means clustering coupled with Genetic algorithm to make online shopping market segmentation [19]. The limitation of the method is that, they use intraclass criteria as a performance metric. But it is unreliable that it is a complete performance measure of clustering based systems. Georgiou and Tsapatsoulis developed a recommendation system by using genetic algorithm based clustering which generates dense clusters that outperforms in terms of accuracy and efficiency [20]. The proposed method is tested in sparse datasets. The creation of the dense cluster is risk in sparse datasets. Genetic Algorithm based clustering takes more computational time.

The limitation of all above methods can be solved by using our proposed method. FCM is used to assign a particular user into more than one cluster and Bat optimization is used as optimizer for initialization of cluster center. Bat optimization is better than other optimization methods like Genetic, Harmony search and particle swarm optimization. Because Bat uses a good combination of major advantages of these algorithms in some way such as frequency tuning. Automatic zooming and parameter control are also other important advantages of Bat optimization. Bat provides very quick convergence at a very initial stage by switching from exploration to exploitation. This creates it an efficient algorithm for clustering [21] when a quick solution is needed. Huang [22] suggested that Bat has global convergence properties and can be suitable for large scale problems. These advantages of Bat improve the clustering result more and also increase the recommendation results. Thus, we concluded that our proposed method can achieve higher efficiency in terms of precision, Recall, MAE and low computation time than other clustering based algorithms.

\section{Proposed methodology}

In this section, we describe at developing a hybrid collaborative fuzzy C-means cluster approach with Bat optimization to improve the prediction accuracy. Fig.1 shows an overview of our proposed approach. The Movie Lens dataset is used for evaluation. Fuzzy Bat Clustering collaborative recommender system is performed in two phases. In the first phase, Fuzzy C Means method clusters the users into different groups based on their past history of ratings. Bat optimization is used to find the optimum cluster Centre points for FCM .It provides better results in optimization than other optimization methods. BA combines with fuzzy was performed well for unsupervised learning [23,24]. In the second phase, the lists of movies are recommended for the active user which might be liked.

\subsection{Fuzzy c means clustering}

FCM is a most widely used clustering technique in which a data point can be a member of different clusters at the same time with different degree of membership. The data point is assigned to clusters based on the distance between the data point and cluster Centre. If the distance of the data point to the cluster center decreases, then the membership of that particular data point to that cluster increases. The distances are computed using Euclidean distance function. The summation of membership of a particular data point to multiple clusters should be equal to one and the range of membership matrix in the range $[0,1]$.After each iteration the membership and the cluster centers value gets updated. FCM is used to cluster $\mathrm{n}$ data points, $X=\left\{X_{1}, \ldots X n\right\}$ into group of c clusters $C=\left\{C_{1}, \ldots . C_{\mathrm{C}}\right\}$.

FCM algorithm consists of the following steps shown below:

1. Membership matrix $M_{\mathrm{ij}}$ is initialized with random values between 0 and 1 from the set of data points randomly.

2. Calculate the fuzzy cluster centers $C_{j}$ using 


$$
C_{j}=\frac{\sum_{i=l}^{N} M_{i j . X_{i}}^{m}}{\sum_{i=l}^{N} M_{i j}^{m}}
$$

3. Calculate the objective function by Using

$$
J_{F C M}=\sum_{I=1}^{N} \quad \sum_{j=1}^{C} \quad M_{i j}^{m}\left\|X_{i}-C_{j}\right\|
$$

$\mathrm{N}$ is the number of data points, $i$ is the particular data point, $j$ is the cluster Centre, $m$ represents fuzzy parameter to control the fuzziness of membership that is greater than 1

4. After each iteration new fuzzy Membership $M_{\mathrm{ij}}$ is updated using

$$
M_{i j}=\frac{1}{\sum_{K=1}^{C}\left(\frac{\left\|X_{i}-C_{j}\right\|}{\left\|X_{i}-C_{k}\right\|}\right)^{\frac{2}{m-1}}}
$$

Where $k$ is the number of clusters, ' $\left\|x_{i}-c_{j}\right\|$ ' is the Euclidean distance between $i^{\text {th }}$ data and $j^{\text {th }}$ cluster Centre .

5. Go to step 2.

6. The iteration will stop when

$$
U^{(k+1)}-U^{(k)}<\xi
$$

Where $\xi$ is a termination criterion. FCM suffers from certain drawbacks. It is sensitive to initialization. Randomly selecting initial cluster centroid may converge to a local minimum. For the sake of overcoming the above drawbacks, we bring Bat Algorithm to avoid local optimum problem of FCM in this paper.

\subsection{Bat Algorithm}

Bat algorithm is the swarm intelligence based Meta heuristic algorithm developed by Xin-She Yang in 2010 [25]. BA is inspired by the echolocation behavior of micro bats with varying loudness and emission of pulse rate. Bat use echoes to find the prey and obstacles. It is used to solve optimization problems in engineering design, classification etc. The optimization algorithm is developed based on the hunting behavior of bats. BA uses a frequency tuning procedure to increase the range of the solutions and also have the capability of automatic zooming to balance the exploration and exploitation. Every bat fly randomly with velocity $V_{i}$ at position $x_{i}$ with a frequency and loudness $A_{i}$. After Bat hunts and finds its prey, then it changes the frequencies, loudness and pulse emission rate $r$. The local Search is strengthened by using a randomization method. The iterations continue until the best solution selected or a certain stop conditions are met. The major steps of the original BA algorithm can be described as the follows.

Initialization: A Population of $\mathrm{d}$ bats (solutions) is initialized $X=\left(x_{1}, x_{2} \ldots, x_{\mathrm{d}}\right)$ randomly and for each solution $x_{i}$, pulse frequency $f_{i}$ is randomly initialized in the range $\left[f_{\min }, f_{\max }\right]$. The pulse rate $r_{i}$ and loudness $A_{i}$ are also initialized, then evaluating this initial populations, and finally, determining the best solution as $X_{\text {best }}$.

Generate the new solution: The new solutions $X_{i}^{(t+1)}$ and velocities $V_{i}^{(t+1)}$ for each solution are given by modifying the frequency, updating the velocities and positions of initial populations (5-7).

$$
\begin{gathered}
f_{i}^{(t)}=f_{\min }+\left(f_{\max }-f_{\min }\right) \cup(0,1) \\
V_{i}^{(t+1)}=V_{i}^{t}+\left(X_{i}^{t}-X_{\text {best }}\right) f_{i}^{t} \\
X_{i}^{(t+1)}=X_{i}^{(t)}+V_{i}^{(t)}
\end{gathered}
$$

Where, $\cup(0,1)$ is a random vector between 0 and 1 from a uniform distribution. $X_{\text {best }}$ indicates the current global best solution after comparing all the solutions of initial $n$ bats. $f i$ is the frequency increment. $f_{\min }$ and $f_{\max }$ values are determined from the size of the problem. For each bat the initial value of frequency is given any value between $f_{\min }$ and $f_{\max }$.

Local search: This search is performed to select a best local solution among the new solutions using the pulse rate probability $r_{i}$ and update the best solution. During the local search, new update solution for each best solution is generated using,

$$
X_{\text {new }}=X_{\text {old }}+\in A^{t}
$$

Where, $\in$ is a scaling factor that controls the convergence of the algorithm. $A^{t}$ is the average loudness of all the bats at the generation.

Evaluate the new solution: Evaluating the new solutions using fitness function.

Accept the best solution: Select the current global best solution under some probability by arranging the solutions. In each generation, the loudness $A_{i}$ and pulse rate $r_{i}$ is updated according to,

$$
A_{i}^{t+1}=a A_{i}^{t}
$$




$$
r_{i}^{t+1}=r_{i}^{0} X(1-\exp (-\gamma)
$$

\subsection{Fuzzy Bat Collaborative Recommender System}

In recent years, cluster-based $\mathrm{CF}$ solves the scalability problems and also increases the quality of recommendation results. The effective clustering method is used to make sure that the users who have the similar interests may belong to one cluster and create the same wavelength (like) neighbors in that cluster. K-Means Clustering is one of the wellknown and normally used clustering approach for huge amounts of data. Wang [26] applied k means clustering and genetic Algorithm (PCA-GAKM) to cluster users that provide higher performance in recommendations. But genetic algorithm has the shortcoming of unguided mutation. Therefore, we used Bat optimization. Komarasamy [27] developed an enhanced $\mathrm{K}$ means clustering technique using the bat optimization. The experimental results from $\mathrm{K}$ means with Bat is good for optimization problems and the author concluded that the proposed algorithm is better than PSO and GA. Fuzzy sets have been commonly used for soft clustering where a individual data can be belong to more than one group with different degrees of membership in the range 0-1.Hence, We use FCM for better clustering instead of strict clustering behavior of $\mathrm{K}$ means. The proposed method proves that the combination of FCM with Bat might give better results than $\mathrm{K}$ means. However, the limitation of FCM is that it is more sensitive to select initial cluster center and stuck in a particular point. Bat Algorithm has been shown to be an effective global metaheuristic optimization method. Khan and Sahari [28] compared the Bat algorithm with other optimization algorithms such as Genetic Algorithm and Particle Swarm Optimization which proves that the BA performs better than others. This has moved to use the bat algorithm with fuzzy C Means clustering for the aim of clustering based recommendation. The process of Fuzzy Bat Collaborative Recommender System is described in Fig.1.

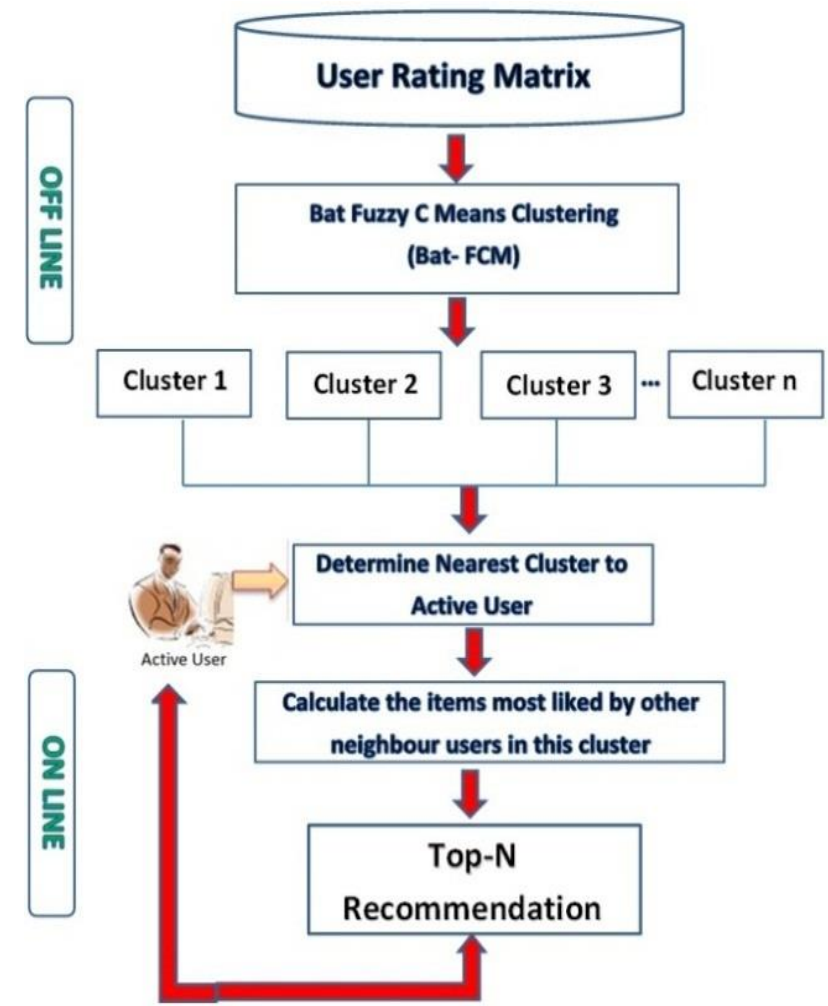

Figure.1 Overview of our proposed collaborative movie Recommender system

Clustering Rating Matrix using Bat fuzzy clustering: To improve the accuracy of recommendation results, Fuzzy C Means clustering method is applied to cluster the Movie Lens Rating Matrix into $k$ number of user clusters. The users in a particular cluster will have similar interests, so that, the movies retrieved by these similar users might be suitable to target user for recommendation. The local optimal problem of FCM is solved by Bat optimization technique. A sample of User item rating matrix of Movie Lens dataset for 5 users on 5 movies has shown in Table 1, where U1-U5 are users and M1-M5 are the items (movies).

The value 1-5 indicates that the rating of likeliness for a particular movie given by user. The value ' 0 ' indicates that, the movie is not rated (not seen) by user. Recommender system finds out the unrated values and recommends top $\mathrm{N}$ movies to user.

Table 1. Example of rating matrix from movie lens dataset

\begin{tabular}{|c|c|c|c|c|c|}
\hline & M1 & M2 & M3 & M4 & M5 \\
\hline U1 & 5 & 3 & 4 & 3 & 3 \\
\hline U2 & 4 & 0 & 0 & 0 & 0 \\
\hline U3 & 0 & 0 & 0 & 0 & 0 \\
\hline U4 & 0 & 0 & 0 & 0 & 0 \\
\hline U5 & 4 & 3 & 0 & 0 & 0 \\
\hline
\end{tabular}


The steps involved in Fuzzy Bat clustering are as follows:

1.Initialize the population size, velocity, frequencies, pulse emission rate, Loudness and the maximum number of iterations.

2.Create an initial population of bats randomly.

3. The membership matrix is initialized with random values between the ranges 0 - 1 .

4. Compute the cluster centers for each bat using (1).

5.Estimate the fitness value of each bat and find the current best solution using (2) \& (3).

6 .For each initial bat solution update the Velocity matrix and the location vector using (5), (6) \& (7).

7.Evaluate the new bat solutions using fitness Function and find the current global best as initial cluster Centre and apply Fuzzy C Means Clustering to cluster the users into different groups.

Nearest Cluster selection: When a current Active user is arrived, Recommender System assigns a particular cluster which has a highest similarity to him/her using Euclidean distance.

Similarity computation: Similarity is calculated between target user and remaining all users in the nearest cluster to calculate the nearest neighbors of the target user. Pearson correlation method is used for similarity computation.

Recommendation: In the process of Recommendation, the movies are recommended to target users that most likely used by other neighbor users which are not seen by him/her. The prediction rating of unrated items for active user is calculated based on weighted average of the rating of items in the same cluster neighbor by using (11) and then make top- $N$ recommendations list to active user.

The rating of unrated movie $i$ for an active user $a$ is predicted by $P a$

$$
P_{a}(i)=\bar{R}_{a}+\frac{\sum_{N \varepsilon C x} \operatorname{Sim}(a, N) \times\left(R_{N}(i)-\bar{R}_{N}\right.}{\sum_{N \varepsilon C x}(|\operatorname{Sim}(a, N)|}
$$

Where,

$a$ - Active User

$\bar{R}_{a}$ - Average of active user $a$

$C x$ - set of nearest neighbors of active user $a$ belonging to one common cluster

$\bar{R}_{N}$ - Average rating score given by active user's neighbor $\mathrm{N}$

$\operatorname{Sim}(a, N)=$ similarity between active user $\boldsymbol{a}$ and Neighbor $\boldsymbol{N}$
Table 2. Predictions for active user

\begin{tabular}{|c|l|c|}
\hline \multirow{5}{*}{ Active User } & $\begin{array}{l}\text { Recommended } \\
\text { Movies }\end{array}$ & Predicted Rating \\
\cline { 2 - 3 } & $\begin{array}{l}\text { Raiders of the } \\
\text { Lost Ark }\end{array}$ & 5 \\
\cline { 2 - 3 } & $\begin{array}{l}\text { Shawshank } \\
\text { Redemption }\end{array}$ & 4 \\
\cline { 2 - 3 } & Fugitive with & 4 \\
\cline { 2 - 3 } & $\begin{array}{l}\text { Dances } \\
\text { Wolves }\end{array}$ & 4 \\
\cline { 2 - 3 } & Rock & 4 \\
\hline
\end{tabular}

\section{Experimental results}

\subsection{Dataset}

To test the recommendation performance, we use a Movie lens dataset [29]. It was collected by the Group lens Research project team at the University of Minnesota for the research work in the field of recommender system. The dataset includes 943 users, 1682 movies and 1,00,000 ratings on a rating scale of 1-5. Every user was rated at least 20 movies and each movie has been rated at least one time. In this dataset, there are three type of information's are available such as demographic information of users, information about movies and rating score of that movies. We use the rating score information for our experiments. We carried out all our experiments on MATLAB2013 Language to simulate the model. The dataset is divided into $80 \%$ as training set and $20 \%$ as testing set. We use the training data to learn a model, and the testing data were used for making predictions. The performance of the proposed fuzzy bat clustering collaborative recommender system is evaluated with existing clustering based CF.

\subsection{Evaluation criteria}

In this paper, we use three metrics to evaluate the accuracy of the proposed recommendation method. The metrics are Mean Absolute Error (MAE), precision, and Recall.

\subsubsection{MAE}

MAE is a Statistical measurement method used to measure the recommendation quality. MAE calculates the deviation between the predicted ratings and actual ratings on test users which is shown in Eq. (12). If the MAE value is small, then the system predicts accurate user ratings. This will provide a good recommendation quality.

$$
M A E=\frac{\sum\left|P_{i j-} a_{i j}\right|}{N}
$$


Where,

$P_{i j}$ - The predicted rating value for user $i$ on item $j$

$N$ - The total number of predicted items

$a_{i j}$ - the real rating of user $\mathrm{i}$ on item $\mathrm{j}$

\subsubsection{Precision}

Precision is defined as the fraction of number of relevant items retrieved by target users to the total number of recommendations. The precision for Top$N$ recommendation is defined in (13).

$$
\text { Precision }=\frac{t_{p}}{t_{p}+f_{p}}
$$

Here,

$t_{p}$ - true positive

$f_{p}-$ false positive

\subsubsection{Recall}

Recall is defined as the fraction of relevant recommended items collected by target user to the total number of items that is actually considered Relevant. The larger recall provides better performance. The Recall for Top- $N$ recommendation is measured by (14).

$$
\text { Recall }=\frac{t_{p}}{t_{p}+f_{n}}
$$

Here,

$f_{n}$ - false negative

\subsection{Performance Results}

In this section, we discuss the results obtained from the proposed method and existing methods. At first the number of clusters are set as 16 for clustering stage. For generating recommendations, we set the number of recommendations top $\mathrm{N}$ for each active user. We compared our proposed system with existing clustering based methods [26] such as PCA-GAKM, PCA-KM, K-Means, UPCC (Pearson correlation coefficient based method) and SOM in terms of MAE, precision, Recall.

MAE was calculated for different number of neighbors by using proposed method and the curve can be plotted in Fig.2. The comparison of existing clustering based CF methods with proposed method regarding MAE is given in Fig.3.As seen in Fig. 3, the proposed fuzzy bat recommender system has a Mean absolute error as 0.62 when the neighborhood size is 25 . On the other hand, existing methods attain MAE above 0.75. All methods reach constant value when the neighborhood is nearby 60 . The traditional UPCC method has a higher MAE and our proposed Fuzzy Bat recommender system has less
MAE value than existing methods. The smaller MAE increases the recommendation accuracy more.

To evaluate the precision of recommendation, the neighbor size was fixed as 25 . The precision of proposed method can be plotted in Fig.4. The precision value was improved and recommends more related movies to target users than other clustering based methods.

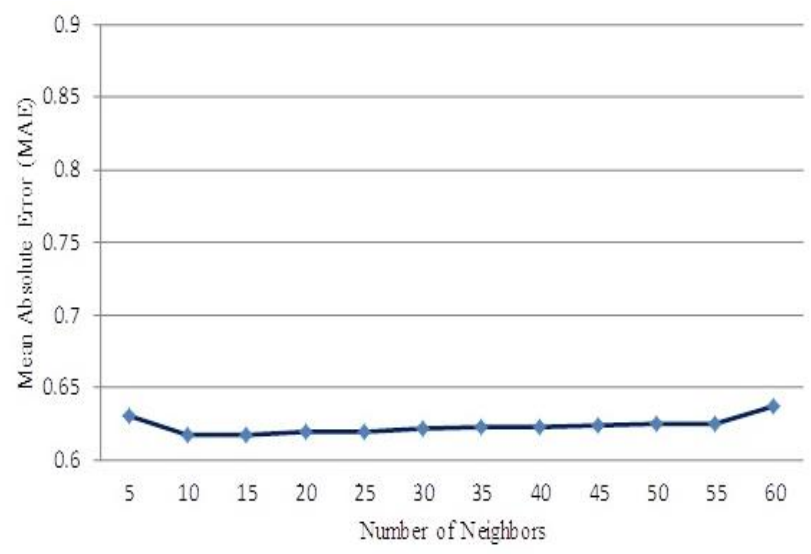

Figure.2 MAE on different number of neighbors using Fuzzy Bat System

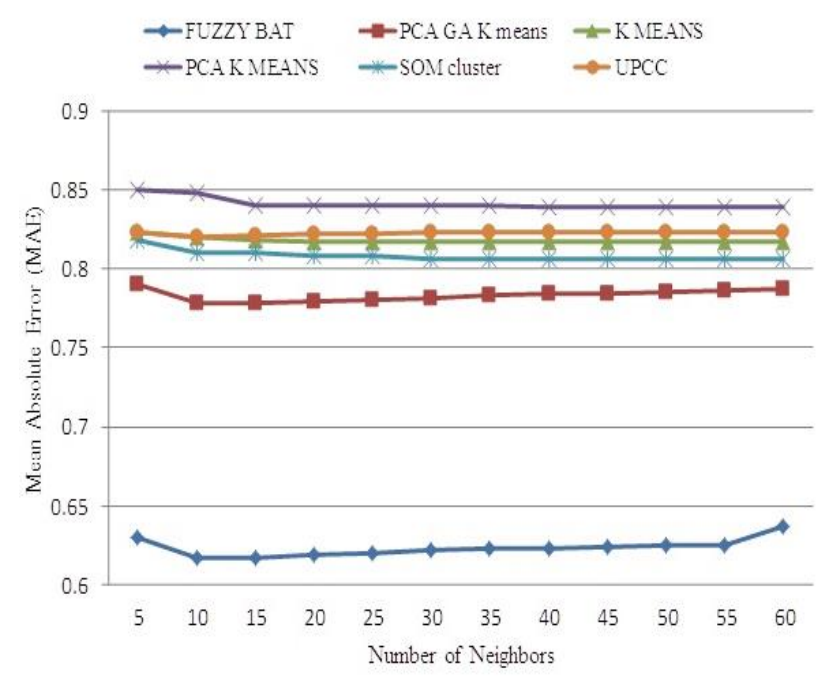

Figure.3 MAE using proposed method and existing clustering methods

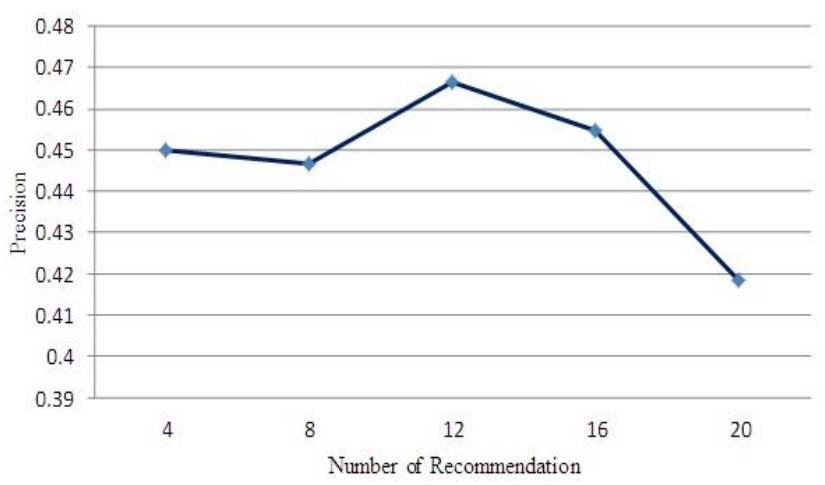

Figure.4 Precision of our proposed method 


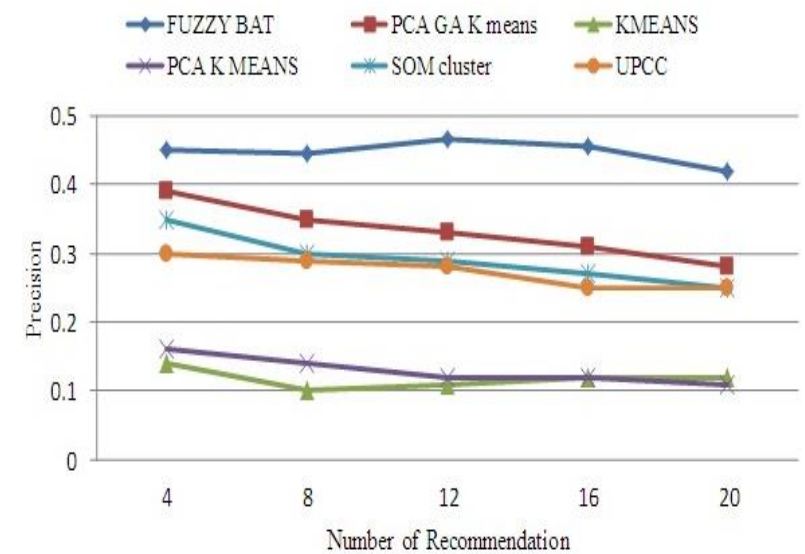

Figure.5 Precision comparison with different number of recommendation

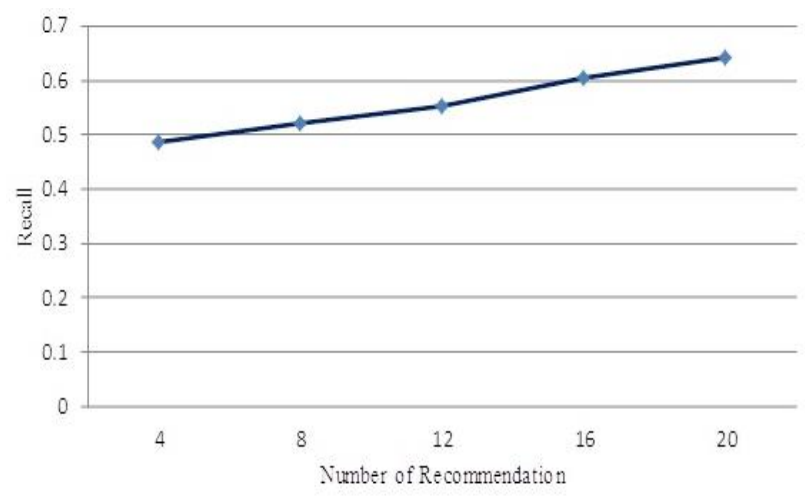

Figure.6 Recall of our proposed method

Fig.5 shows that our Fuzzy Bat Recommender system provides high precision rate than existing clustering based recommender systems. Our approach achieves maximum precision value 0.45 than existing methods when number of recommendation is 4 .

The Recall of proposed method can be presented in Fig.6. This explains that Fuzzy Bat Recommender system provides greater recall value according to different number of recommendations. In this figure the recall increases continually when the number of recommendation increases. This confirm that the proposed system provide improved predictions. The Recall comparison with existing methods was shown in Fig. 7. From this figure, our approach achieves maximum Recall of 0.64 when number of recommendation is 20 . The existing methods achieve less precision than our proposed method.

The precision and recall measures are conflict. The Precision was decreased when the number of recommendations increased. But the Recall value increases when number of recommendations increases. From the above experimental results, it is evident that our proposed method produces good results regarding MAE, precision and recall than other existing methods.

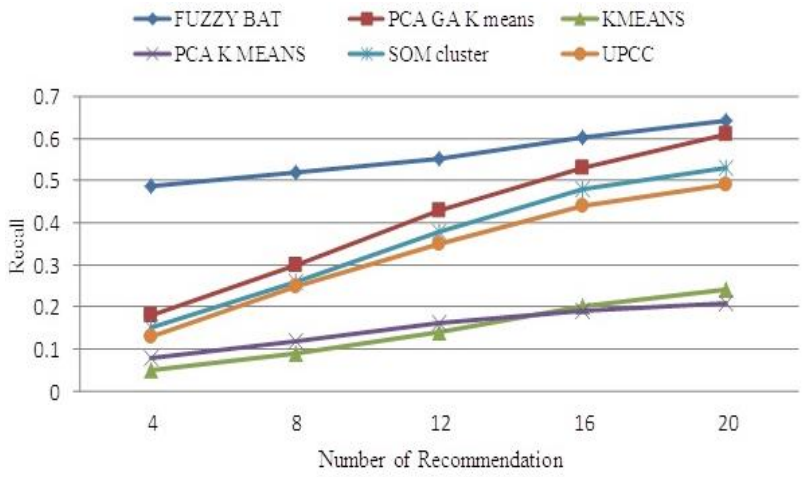

Figure.7 Recall comparison with existing cluster methods

\section{Conclusion}

In this paper, we developed a Collaborative filtering method to suggest movie recommendations for e-commerce Customers. Collaborative Filtering $(\mathrm{CF})$ is a popular method for making recommendations in internet. Data sparsity and scalability are the two main problems of traditional $\mathrm{CF}$ algorithms. In this article, we have presented a hybrid model based collaborative filtering approach to reduce the above said problems. At first the users are clustered using FCM based on the similarity of user's rating. Bat algorithm is used as optimizer which gives initial cluster Centre for FCM. Bat uses frequency tuning, automatic zooming and parameter control properties. These properties help to find out a good optimal cluster Centre quickly and also improve the clustering performance. Secondly, the nearest neighbors of active user can be found by Pearson correlation method. Finally the recommendations are made by using the neighbors of active user. The performance was analyzed on movie Lens dataset and the results of proposed method were satisfactory when compared with other algorithms. The simulation results shows that our proposed method has less MAE and increased Precision and Recall value. This outperforms the performance regarding prediction accuracy and also capable of providing reliable and more personalized movie recommendation systems. For future work, we can consider other features as trust, contextual and demographic information.

\section{References}

[1] J.L.Herlocker, J.A.Konstan, A.Borches and J.Riedl, "An algorithmic framework for performing collaborative filtering", In: Proc. of the $22^{\text {nd }}$ Annual International ACM SIGIR Conference on Research and Development in Information Retrieval, Aug.15, Berkeley, California, ACM press, pp.230-237, 1999. 
[2] E.Adomavicius and A.Tuzhilin, "Toward the next generation of recommender systems: a survey of the state of the art and possible extensions", IEEE Transactions on Knowledge and Data Engineering, Vol.17, issue 6, pp.734749, 2005.

[3] J.L.Sanchez, F.Serradilla, E.Martinez, and J.Bobadilla, "Choice of metrics used in collaborative filtering and their impact on recommender systems", In: Proc. of the IEEE International Conference on Digital Ecosystems and Technologies DEST, pp. 432-436, 2008.

[4] G.Ulrike and F.R.Daniel. "Persuasion in recommender systems." International Journal of Electronic Commerce, Vol.11, No.2, pp.81-100, 2006.

[5] C.N.Ziegler, S.M.McNee, J.A.Konstan, and G.Lausen, "Improving recommendation lists through topic diversification", In: Proc. of the 14th international conference on World Wide Web; pp.22-32, 2005.

[6] L.Landau, "An Introduction to Recommender Systems", Cambridge University Press, New York, 2011.

[7] L.H.Letizia, "An agent that assists web browsing", In: Proc. of the 1995 international joint conference on artificial intelligence, Montreal, Canada, p.924-9, 1995.

[8] J.B.Schafer, D.Frankowski, J.Herlocker, and S.Sen, "Collaborative filtering recommender systems", The Adaptive Web, LNCS 4321. Berlin Heidelberg (Germany): Springer; pp.291-324, 2007.

[9] R.Burke, "Hybrid web recommender systems The Adaptive Web, Springer-Verlag, Berlin Heidelberg", P.Brusilovsky, A.Kobsa, W. Nejdl (Eds.), pp. 377-408, 2007.

[10] B.Sarwar, G.Karypis, J.Konstan, and J.Riedl, "Item-based collaborative filtering recommendation algorithms", In: Proc. of the 10th Int. Conf. World Wide Web, pp.285-295, May 2001.

[11] F.O.Isinkaye, Y.O.Folajimi, and B.A.Ojokoh, "Recommender system: Principles, methods and evaluation", Egyptian Informatics Journal, pp.261-273, 2015.

[12] C.Huang and J.Yin, "Effective association clusters filtering to cold-start recommendations", In: Proc. of the Seventh international conference on fuzzy systems and knowledge discovery (FSKD), Vol.5, pp.24612464, 2010.

[13] N.Pal and J.Bezdek, "On cluster validity for the fuzzy C-means model", IEEE Transactions on Fuzzy Systems, Vol.3, No.3, pp.370-379, 1995.
[14] D.Goldberg, D.Nichols, B.M.Oki, and D.Terry, "Using collaborative filtering to weave information tapestry", Commun. ACM 35 Vol.12, pp.61-70, 1992.

[15] C.Birtolo and D.Ronca, "Advances in Clustering Collaborative Filtering by means of Fuzzy C-means and trust", Expert Systems with Applications, Vol.40, pp.6997-7009, 2013.

[16] K.Treerattanapitak and C.Jaruskulchai, "Exponential Fuzzy C means for collaborative filtering", Journal of computer science and technology, Vol.27, No.3, pp.567-576, 2012.

[17] H.Koohi and K.Kiani, "User based Collaborative Filtering using fuzzy C-means clustering", Measurement 91, pp.134-139, 2016.

[18] Q.Li, and B.M.Kim, "Clustering approach for hybrid recommendation system", In: Proc. of the International Conference on Web Intelligence, Halifax, Canada, Vol.9, pp.33-38, 2003.

[19] K.Kim, and H.Ahn, "A recommender system using GA K-means clustering in an online shopping market", Expert System with Application, Vol.34, No.2, pp.1200-1209, 2008.

[20] O.Georgiou, and N.Tsapatsoulis, "Improving the scalability of recommender systems by clustering using genetic algorithms", In: Proc. of the $20^{\text {th }}$ International conference on Artificial Neural Networks, Thessaloniki, Greece, pp.442-449, 2010.

[21] X.S.Yang, "Bat Algorithm: Literature Review and Applications", International Journal of Bio-Inspired Computation, Vol. 5, No. 3, pp. 141-149, 2013.

[22] G.Q.Huang, W.J.Zhao, and Q.Q.Lu, "Bat algorithm with global convergence for solving large-scale optimization problem", Application Research of Computers, Vol. 30, No. 3, pp.1-10, 2013.

[23] X.S.Yang and A.H. Gandomi, "Bat Algorithm: A Novel Approach for Global Engineering Optimization", Engineering Computations, Vol.29, No.5, 2012.

[24] K.Khan, and A.Sahai, "A fuzzy c-means bisonar-based Metaheuristic Optimization Algorithm", International Journal of Interactive Multimedia and Artificial Intelligence, Vol.1, no.7, pp.26-32, 2012.

[25] X.S.Yang, "A New Metaheuristic BatInspired Algorithm", Nature Inspired Cooperative Strategies for Optimization, Studies in Computational Intelligence, Vol.284, pp.65-74, 2010. 
[26] Z.Wang, X.Yu, N.Feng, and Z.Wang, “An improved collaborative movie recommendation system using computational intelligence", Journal of Vis Lang Computer, Vol.25, pp.667$75,2004$.

[27] G.Komarasamy and Amitabh Wahi, "An Optimized K-means Clustering Technique using Bat Algorithm", European Journal of Scientific Research, Vol.84, No.2, pp.263-273, 2012.

[28] K.Khan, and A.Sahai, "A comparison of BA, GA, PSO, BP and LM for training feed forward neural networks in e-learning context", International Journal of Intelligent Systems and Applications (IJISA), Vol.4, No.7, pp.23-29, 2012.

[29] http://grouplens.org/datasets/movielens/ 100k/ 